\title{
Foam Level Control in a Water Model of the LD Converter Process
}

\author{
Wolfgang Birk, and Alexander Medvedev \\ Control Engineering Group, Luleå University of Technology, SE-971 87 Luleå, Sweden
}

\begin{abstract}
This paper deals with estimation and control of foam level in dynamic foaming. An improved foam level estimation methodology from a microphone signal and its automatic calibration is presented. The dynamical reaction of the foam level on air lance movements is modelled using system identification. Based on the resulting mathematical model, a controller for foam level stabilisation is designed and applied to a water model, representing the LD converter process. It is shown that the foam level can be controlled using a microphone as the measurement device and air lance movement as the actuator.
\end{abstract}

\section{INTRODUCTION}

Steel is widely produced from hot metal and scrap. Hot metal with about $4-5 \% C$ is produced from iron oxide pellets and coke by the blast furnace. After the blast furnace, the hot metal is poured into a ladle, sulfur is removed from the hot metal by a reaction with lime. Thereafter, the hot metal can be converted into steel by a top blown basic oxygen furnace (BOF). Oxygen is supplied through a lance from above and is jetted onto the metal bath at supersonic speed. Scrap, slag forming agents and hot metal are charged to the vessel and, thereafter, the lance is lowered with oxygen blown through. The oxygen jet forms a cavity at the bath surface where $F e, S i, M n$ and $C$ are oxidised. Metal droplets are splashed and mixed together with the slag. Carbon dissolved in the metal phase reacts with $\mathrm{FeO}$ to form carbon monoxide.

This means that a foam containing metal droplets, metal oxides (slag) and $C O$ bubbles is formed. The effective surface area of this foam is large and thereby the chemical reactions involved in this process are fast. To make this process effective, a large foam volume is needed. However, if the foam level is too high, slopping occurs causing reduction in metal exchange and environmental pollution. The formation of the foam is manually controlled by adjusting the oxygen lance level. The foam level in the vessel is nearly impossible to measure, but there has been somewhat successful methods using radio waves and sonic meters. Optical methods based on laser technology have been tried with bad results because of the strong dust formation during the foaming.

A very simple and continuous method to estimate foam level is to use a microphone and measure the sound intensity at certain frequency bands. As the foam level increases, the sound intensity decreases. Microphones or sonic meters have been widely used since 1970 in many steel plants. However, the sound signal generated is seldom used in order to automatically control the foam level. Piombino Steel shop, ILVA Taranto and British steel are some examples where the sound signal is used as control signal [1], [2], [3], but not for dynamic control, as continuous lance movements are not supported by the actuator and slag properties are often unknown.

In [4], the sonic meter signal is estimated from off-gas flow rate and $C O$ content in the gas. There, the deviation of estimated signal from measured signal is used to detect slopping, combining sonic meter and gas-analysis.
In order to study the process water models are used. The disadvantage with water models are that only a few parameters of the real high temperature process can be studied. In the case of the study of foam level control in the LDprocess it was felt to use a water model with large height to diameter ratio. For a detailed descriptions of the water model see [5]. Moreover, a brief introduction to foaming and first results on foam level estimation are given there.

The purpose of the present work is to improve the formerly introduced foam level estimate algorithm and supply an automatic strategy to calibrate the estimation algorithm. Another goal is, to demonstrate the possibility of closed loop control of the foam level in the water model using the sound signal as measurement. Foam level control in a LDconverter can be used to automatically avoid slopping.

\section{FOAM LEVEL ESTIMATION}

In [5] an estimation algorithm for the foam level based on a microphone signal is derived. There, the foam level is computed from the short time sound spectra as

$$
\hat{h}(t, \omega)=\frac{\ln \left(I_{0}(\omega)\right)-\ln (I(h(t), \omega))}{\hat{\beta}_{F}(\omega)},
$$

where $I_{0}(\omega)$ is the magnitude of the sound spectra without foam and $I(h(t), \omega)$ is the magnitude of the sound spectra at different foam levels. Furthermore, $\hat{\beta}_{F}(\omega)$ is the attenuation coefficient, which is frequency dependent.

In the sequel, the following fluids are used in the foaming experiments

\begin{tabular}{c|c|c|c} 
Fluid & $\begin{array}{c}\text { Viscosity } \\
{[\mathrm{Cp}]}\end{array}$ & $\begin{array}{c}\text { Foam life } \tau \\
{[\mathrm{s}]}\end{array}$ & $\begin{array}{c}\text { Surface tension } \\
{[\mathrm{N} / \mathrm{m}]}\end{array}$ \\
\hline $\mathrm{A}$ & 3.1 & 3448 & 0.27 \\
\hline $\mathrm{B}$ & 1.0 & 189 & 0.49
\end{tabular}

During the design of the foam level estimation, data from a foaming experiment with fluid A are used. Under the experiments the sound signal from the microphone is logged and the foam level is manually read off. The data set is used in the subsequent three subsections and all plots refer to this data set.

Foam generated from fluid A has a higher stiffness and better sound damping capabilities compared to fluid B, as fluid B generates foam faster than fluid A. These contradictory characteristics will be exploited to validate the estimation algorithm.

\section{A. Improved estimation}

As the sound spectra are estimated from sampled data, the accuracy of the estimate directly affects the foam level estimate. According to [6], the confidence interval in $d B$ for the sound spectra estimate of $M$ frequency bands from a sequence of $N$ samples is given by

$$
R_{x} \approx K\left(\frac{N}{M}-0.833\right)^{-0.5}
$$

where 


\begin{tabular}{l|l|l|l|l}
$x$ & $80 \%$ & $90 \%$ & $96 \%$ & $98 \%$ \\
\hline$K$ & 11.2 & 14.1 & 17.7 & 20.5
\end{tabular}

Hence, frequency resolution has to be traded off against confidence interval for the estimate. The formerly presented estimation scheme is continuously calculating an estimate every second with a frequency resolution of $1 \mathrm{~Hz}$, which means $N$ is equal to $M$ and gives a confidence interval of $R_{90 \%} \approx 34.5 d B$. By increasing the frequency resolution from $1 \mathrm{~Hz}$ to $10 \mathrm{~Hz}$, the confidence interval can be narrowed to $R_{90 \%} \approx 3.2 \mathrm{~dB}$. Consequently, the foam level estimate can be improved by reducing the frequency resolution.

Moreover, the power spectral density (PSD) is a better measure than magnitude of the Fourier transform as it is based on the power of the signal, which is always bounded, and in contrary to the Fourier transform, does not depend on the stochastic properties of the sound signal.

Consequently, the estimation algorithm has to be reformulated using the PSD. Experiments have shown that the dynamic behaviour of the foam is rather slow and the estimation frequency $f_{P S D}$ is set to $1 \mathrm{~Hz}$. Between the estimation instances, the sound signal is sampled at the much higher frequency $f_{s}$, which is chosen according to the microphone performance and the sound signal content. Logged sound signals from foam experiments are analysed and a sampling frequency $f_{s}=8 \mathrm{kHz}$ appeared to be sufficient.

The PSD of the sound source can be computed as the Fourier transform of the autocorrelation function of the sound sequence between the estimation instances. While $N$ is given by $f_{s} / f_{P S D}$, the number of frequency bands $M$ has to be chosen. Here, $M$ is chosen as $N / 20=400$ which corresponds to a frequency resolution of $10 \mathrm{~Hz}$. Thus, the confidence interval becomes $R_{90 \%} \approx 3.2 \mathrm{~dB}$.

The sampled autocorrelation function of the sound sequence is given by

$$
\begin{aligned}
\phi(t, m) & =\frac{1}{N-m} \sum_{n=0}^{N-m-1} S_{n} S_{n+m} \\
m & =0,1, \ldots, M
\end{aligned}
$$

where $S_{i}$ are the samples and $t$ is the time instance when the PSD is computed. By applying the Fourier transform to (3), the PSD is found

$$
\begin{aligned}
\Phi\left(t, \omega_{n}\right) & =\frac{1}{f_{s}}\left[\phi(t, 0)+2 \sum_{m=1}^{M} \phi(t, m) x_{m} \cos \frac{m n \pi}{M}\right](4) \\
x_{m} & =\frac{1}{2}\left[1+\cos \frac{m \pi}{M}\right] ; m=0,1, \ldots, M \\
\omega_{n} & =\frac{f_{s} n \pi}{M} \\
n & =0,1, \ldots, M-1
\end{aligned}
$$

Here, $x_{m}$ is the Hanning window, which is used to smooth the spectra. Fig. 1 shows the estimated power spectral density of the design data set.

Accordingly, (1) can be modified to use the PSD instead of the magnitude of the Fourier transform

$$
\hat{h}(t, \omega)=\frac{\ln \left(\Phi_{0}\left(\omega_{n}\right)\right)-\ln \left(\Phi\left(t, \omega_{n}\right)\right)}{\hat{\beta}_{F}\left(\omega_{n}\right)}
$$

\section{B. Automatic calibration}

Several parameters have to be calibrated in the estimation algorithm. The parameters $\beta_{F}$ and $\Phi_{0}$ have to be

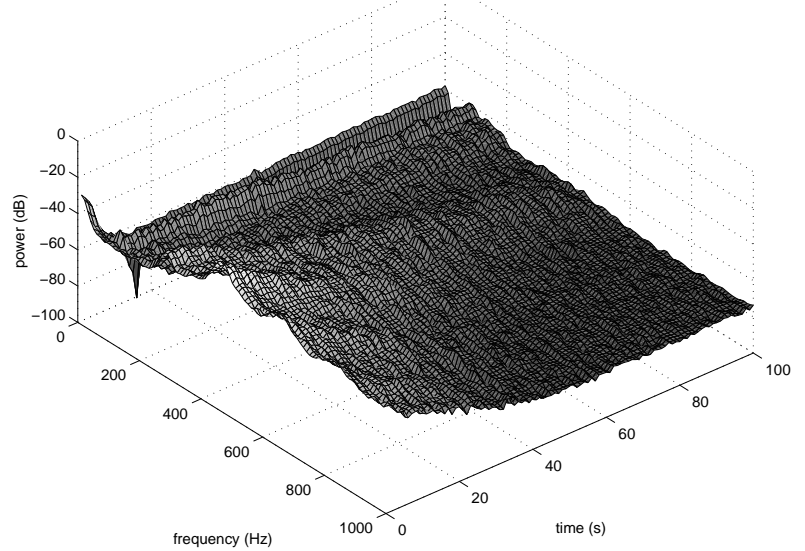

Fig. 1. Power spectral density of the design data set (fluid A)

identified and the frequency bands which fit best should be chosen. To facilitate calibration, an automatic calibration scheme has to be available, which provides the user with candidate frequencies and a parameter set for each frequency.

Since there is no automatic procedure to measure the foam level, time marked manually observed foam levels have to be used for the calibration. In the water model, foam level can be easily read using a ruler, which is attached to the water model. Then, the observed foam level points are interpolated over time using cubic splines and sampled at the same frequency as $\Phi$ is available.

Assuming that $\Phi$ and the observed foam level $h$ are available as a time series with $p$ points, then for each frequency $\omega_{n}$ fixed, $\ln \left(\Phi\left(t, \omega_{n}\right)\right)$ can be fitted to $h(t)$ in a least squares sense. Rewriting (5) to

$$
\hat{h}\left(t, \omega_{n}\right)=\underbrace{-\frac{1}{\beta\left(\omega_{n}\right)}}_{\theta_{1}} \ln \left(\Phi\left(t, \omega_{n}\right)\right)+\underbrace{\frac{\ln \left(\Phi_{0}\left(\omega_{n}\right)\right)}{\beta\left(\omega_{n}\right)}}_{\theta_{2}}
$$

the following regressor can be set up for each $\omega_{n}$

$$
\left[\begin{array}{c}
h\left(t_{1}\right) \\
\vdots \\
h\left(t_{p}\right)
\end{array}\right]=\left[\begin{array}{cc}
\ln \left(\Phi\left(t_{1}, \omega_{n}\right)\right) & 1 \\
\vdots & \vdots \\
\ln \left(\Phi\left(t_{p}, \omega_{n}\right)\right) & 1
\end{array}\right]\left[\begin{array}{c}
\theta_{1} \\
\theta_{2}
\end{array}\right]
$$

and the least squares estimate for parameters $\theta_{1}$ and $\theta_{2}$ is given by

$$
\left[\begin{array}{c}
\hat{\theta}_{1} \\
\hat{\theta}_{2}
\end{array}\right]=\left[\begin{array}{cc}
\ln \left(\Phi\left(t_{1}, \omega_{n}\right)\right) & 1 \\
\vdots & \vdots \\
\ln \left(\Phi\left(t_{p}, \omega_{n}\right)\right) & 1
\end{array}\right]^{\dagger}\left[\begin{array}{c}
h\left(t_{1}\right) \\
\vdots \\
h\left(t_{p}\right)
\end{array}\right],
$$

where $\dagger$ denotes the pseudo-inverse.

Evaluating the standard deviation of the estimation error $\epsilon$, frequencies for foam level estimation can be chosen. Since $\epsilon\left(\omega_{n}\right)$ characterizes the sound attenuation behaviour of the foam, a finger print of the foam is found, Fig. 2.

Fig. 3 shows the estimated foam level using the best fitting frequency. There, $\epsilon$ is $17.9 \mathrm{~mm}$ and follows rather precisely the confidence interval of the estimate, which becomes $\pm 19.5 \mathrm{~mm}$ for the used frequency.

\section{Optimal weighted estimate}

Since several frequencies can give good fits for the foam level estimation, the weighted sum of those frequencies in- 


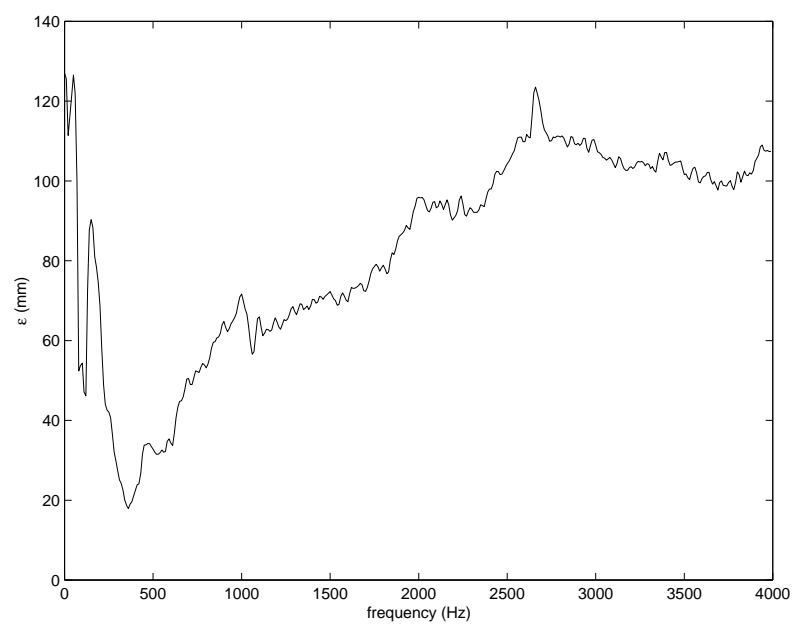

Fig. 2. Plot of $\epsilon$ (Finger print of the foam) for fluid A

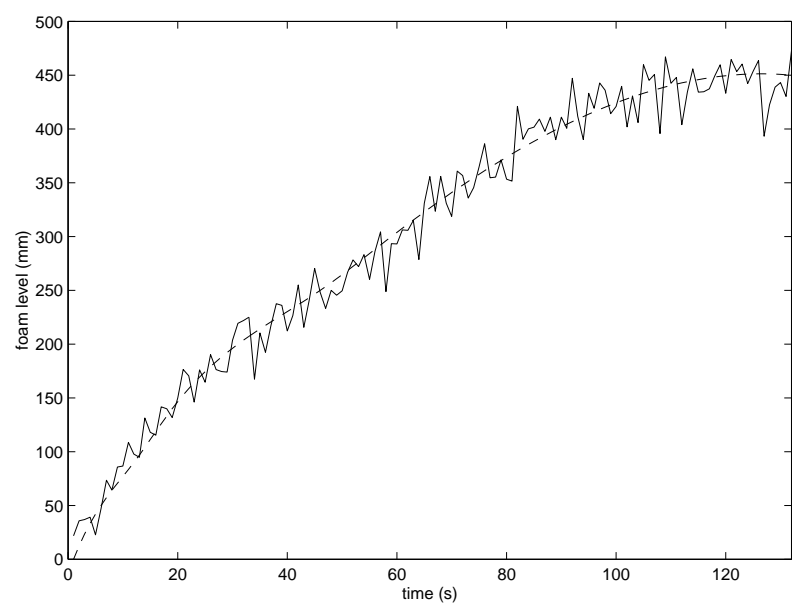

Fig. 3. Foam level estimated (solid) versus observed foam level (dashed) using the best fitting frequency band (fluid A).

troduces redundancy and robustness against changes of the foam characteristics.

In order to find the optimal weighted estimate, which even includes the optimal number of frequencies to use, the set of frequencies is re-ordered according to their ability to fit the observed foam level. As ordering measure $\epsilon\left(\omega_{n}\right)$ is used. The ordered set is denoted $\mathcal{F}$ and $\mathcal{F}_{i}$ denotes the $i^{t h}$ best fitting frequency.

Using the weighting scheme

$$
w\left(\omega_{n}\right)=\frac{1}{\operatorname{std}\left(\epsilon\left(\omega_{n}\right)\right)}
$$

the weighted foam level estimate composed of $r$ frequencies is given by

$$
\hat{h}_{r}(t)=\frac{1}{\sum_{i=1}^{r} w\left(\mathcal{F}_{i}\right)} \sum_{i=1}^{r} w\left(\mathcal{F}_{i}\right) \hat{h}\left(t, \mathcal{F}_{i}\right)
$$

Deriving the standard deviation of the estimation error for the weighted estimate as above and denoting it $\xi(r)$, an optimality measure for the weighted estimate is found. Hence, searching for $\min _{1 \leq i \leq M} \xi(i)$ the best choice for $r$ is obtained.

Consequently, the optimal weighted estimate is given by $\hat{h}_{r}(t)$ for which $\xi(r)=\min _{1<i<M} \xi(i)$. Fig. 4 shows a plot for $\xi$. Obviously, the optimal number of frequencies to use

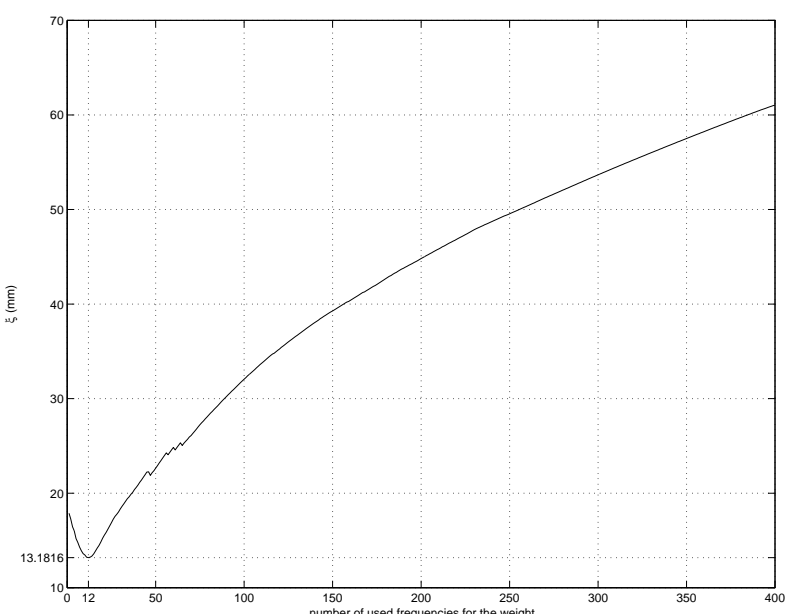

Fig. 4. Plot of $\xi$ (Optimality measure) for fluid A

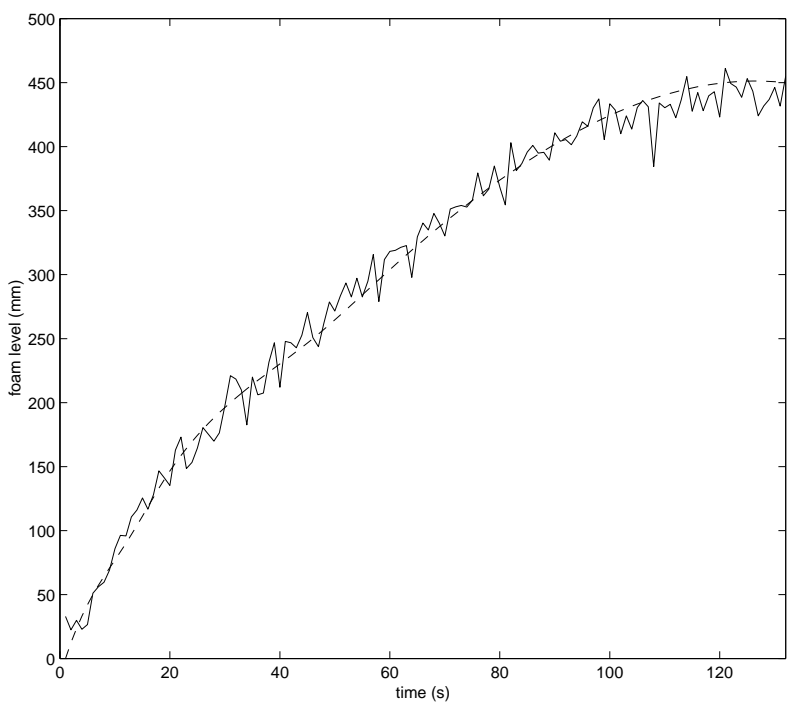

Fig. 5. Optimal weighted foam level estimate for fluid A

is 12 , which reduces the standard deviation of the estimation error from $17.9 \mathrm{~mm}$ down to $13.2 \mathrm{~mm}$. The resulting optimal weighted estimate is shown in Fig. 5.

\section{Validation}

In order to validate the estimation, a different fluid with less attenuation capability is used (fluid B). Furthermore, the generated foam has a shorter foam life $\tau$ [5] and will break down considerably faster than the foam generated from fluid A.

Since, the accuracy of the estimation directly depends on the attenuation capability of the foam. Hence, reliability of the estimation concept is tested. Fig. 6 shows the results from the application of the estimation concept. Obviously, the performance is reduced for low and high foam levels. Still, the estimation is reasonably good although the prerequisites are worse.

\section{FOAM LEVEL CONTROL}

According to [5], there are several potential actuators to control the foam level in the LD converter. Since the gasflows to the converter should be constant, their usage as actuator would cause major changes in the converter process.

Hence, the position of the oxygen lance appears to be the 


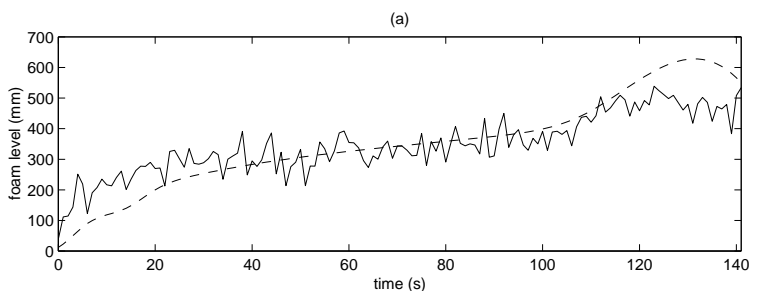

(b)

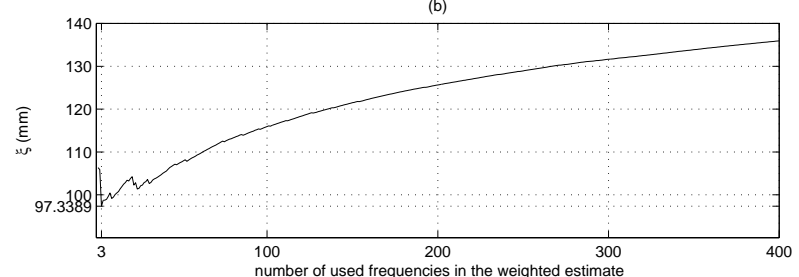

Fig. 6. Estimation with fluid B. (a) Estimated foam level (solid) versus observed foam level (dashed) using the optimal weighted estimate. (b) Optimality measure $\xi$

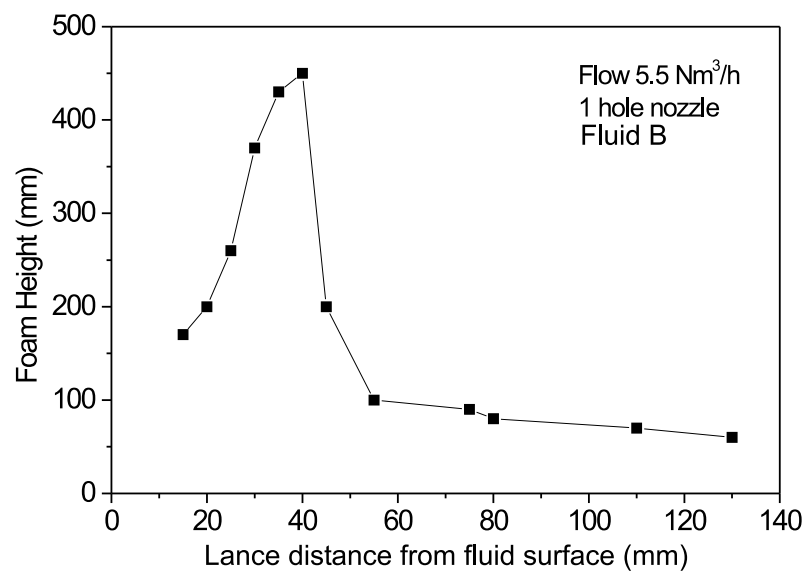

Fig. 7. Dependency of steady state foam level on the lance nozzle distance from fluid surface

natural choice. In manually operated foaming experiments in the water model the foam level could be affected by lance movements.

Thus, a series of foaming experiments has been conducted, where the lance position is only changed between the experiments. In each experiment the steady state value of the foam level is read off.

Fig. 7 shows the lance position versus the steady state value of the foam level. Apparently, the curve reflects the DC-gain from the lance position to the foam level. Important to notice are two areas: below and above $40 \mathrm{~mm}$. At $40 \mathrm{~mm}$ a sign change of the DC-gain occurs, and thus actuator movement in the two areas result in different impact on the foam. Additionally, the area below $40 \mathrm{~mm}$ is very narrow, which can lead to prevalent saturation.

\section{A. Identification of the foam dynamics}

From the static foam experiments it can be expected that the foam dynamics can be approximated by a linear model for the area above $40 \mathrm{~mm}$. For the use in the controller design, a linear mathematical model is identified from inputoutput data. During a foaming experiment, a pseudo random noise sequence is send to the lance actuator and the resulting estimated foam level is recorded.

As the lance movement has velocity saturation and the dynamical behaviour of the foam is rather slow, the sampling time is chosen to $2 \mathrm{~s}$. Hand driven foam control trials
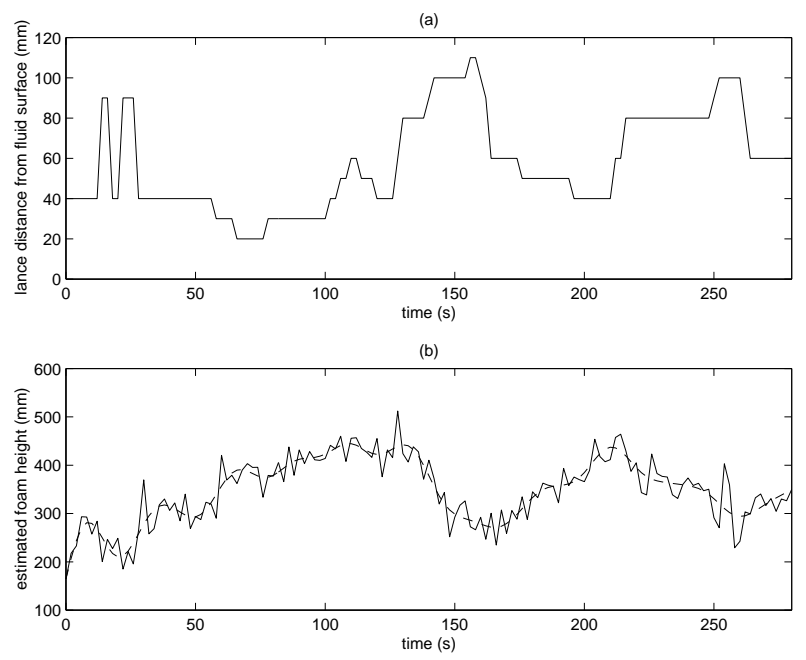

Fig. 8. Identification data. (a) Pseudo random noise as actuator movement. (b) Estimated foam level (solid), filtered version (dashed).

have shown that the sampling time for control purposes can be increased to around $6 \mathrm{~s}$.

Fig. 8 shows input and output signal. There, it can be observed that increasing the distance from the fluid surface does reduce the foam level.

Using the subspace identification algorithm n4sid [7], a linear state space model of order 4 appeared to perform well on the recorded data set. The resulting model will be denoted $G(q)$, where $q$ represents the backward shift operator.

\section{B. Design and analysis}

In a first attempt to control the foam level, a PI controller in a one degree of freedom control configuration is designed. For this end, an iterative design process is used, where the closed-loop poles are placed so that the output sensitivity function of the closed-loop does not exhibit peaks larger than two. The output sensitivity function, which is the transfer function from output disturbance to output signal, is given by

$$
S(q)=\frac{1}{1+G(q) C(q)}
$$

where $C(q)$ denotes the controller. The sensitivity function specification can be written as

$$
M_{S}=\sup _{0 \leq \omega \leq \pi / T_{s}}\left|S\left(\exp \left(-j \omega T_{s}\right)\right)\right| \leq 2
$$

It can be shown that the relative stability margin $M_{S}$ yields a gain margin of at least 2 and a phase margin of more than 30 degrees. Furthermore, plant perturbations of less than 0.5 in magnitude will not affect closed loop stability.

Using the linear model $G(q)$ from the identification, the PI controller $C(q)$ is designed via the root-locus method. The resulting sensitivity function $S(q)$ exhibits a small peak, Fig. 9. Accordingly, the closed loop system is robustly stable for even rather large uncertainties, [8].

Since the movement of the lance has to be restricted to the area above $40 \mathrm{~mm}$, saturation phenomena have to be considered. In order, to prevent integrator wind-up, the PI controller has to be implemented with an anti-windup scheme. The designed closed loop system is depicted in Fig. 10. 

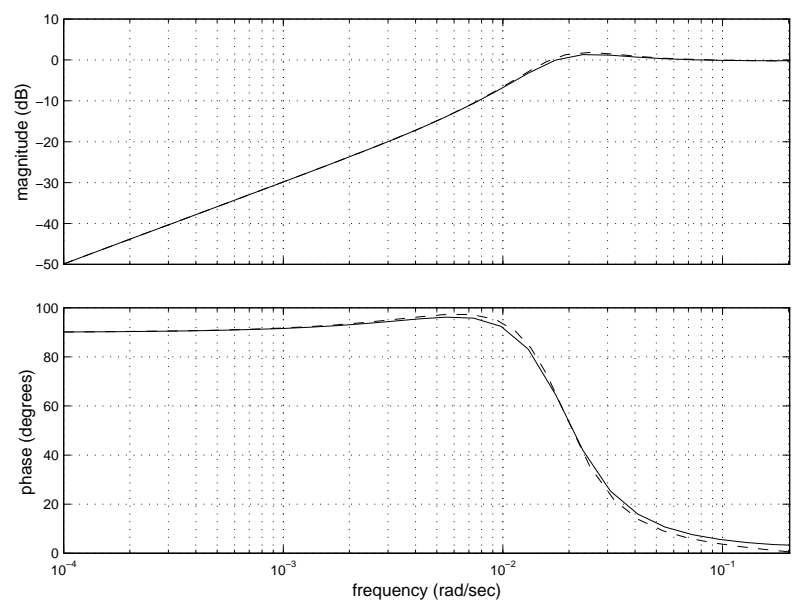

Fig. 9. Bode plot of the output sensitivity function of the closed loop system. Sample time $2 s$ (solid), sample time $6 s$ (dashed).

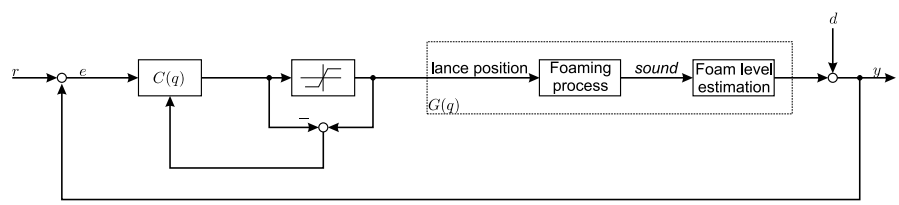

Fig. 10. Block diagram of the closed loop system with PI controller and anti-windup.

\section{EXPERIMENTS}

Both foam level estimation and controller are implemented on the DSP of the water model. The control loop is running at a sampling time of $6 s$, while the estimation algorithm is run with $2 \mathrm{~s}$.

The reference value during a control experiment could be changed via the user interface. Hence, arbitrarily chosen fixed reference values could be tested and transient behaviour observed. Fig. 11 shows the performance of the closed loop system after a reference value change. It can be seen that the controller is able to drive the control error to zero. The standard deviation of the control error in the displayed range accounts for up to $46 \mathrm{~mm}$.

During the control experiments spot tests of the foam level are taken. A bias between estimated and observed foam height appears, which is a result of the changes in the

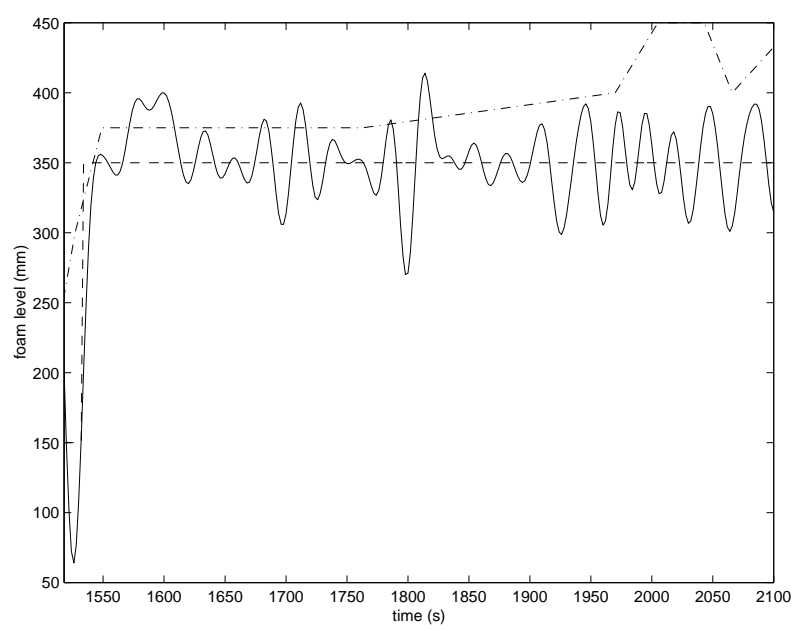

Fig. 11. Performance of the closed loop system with fluid A. Foam level reference value (dashed), Filtered foam level estimate (solid), Observed foam level (dashed-dotted). lance position. Naturally, the lance position should affect the foam level estimation, as the sound source is moving with the position changes of the lance.

\section{Conclusions}

The estimation of foam level in a water model of an LD converter process from microphone signals is discussed. It is shown how the estimation algorithm can be automatically calibrated. The estimation concept is applied to foaming experiments which are conducted in the water model. Tests with different fluids are performed and prove that the estimation scheme can cope with a wide range of fluids.

Furthermore, the estimated foam level is then used as a measurement signal for closed loop control of the foam level using a PI controller. Control experiments are conducted in the water model and indicate that foam level control using lance positioning is possible. Still, it has to be analysed how dynamical changes in the lance position affect the foam level estimation, since a bias appeared during the control experiments.

In order to be able to apply closed loop control of the foam level in the LD converter the following issues have to be discussed. Firstly, the experiments with the water model do not reflect how properties of the chemical reaction or the slag influence estimation and control of the foam level. Therefore, a high temperature model of the LD-converter should be used to validate both estimation and control. Secondly, most converters are not equipped with lance actuators that can provide continuous motion of the lance. Thus, the LD-converter lance actuator has to be changed in order to be usable for dynamic foam level control.

Despite these portability issues, dynamic control of the foam level has the potential of preventing slopping in the LD-converter process. Finally, if applied in combination with carbon content estimation, [9], automatic control of the LD-converter process is possible.

\section{REFERENCES}

[1] C. Bencini and A. Poli, "Automation for refining and slag control in LD process at AFP/Piombino Steel Shop," in Steelmaking Conference Proceedings, pp. 193-198, 1993.

[2] A. Abbatangelo, M. D. Rena, M. Palchetti, and L. Zampetti, "Blowing pattern computerised control at Taranto Steelshop," in Steelmaking Conference Proceedings, pp. 337-342, 1990.

[3] D. Anderson, C. M. Barnes, and H. J. Whittaker, "Fully dynamic process control of the BOS in British Steel," in Steelmaking Conference Proceedings, pp. 379-387, 1991.

[4] D. Widlund, A. Medvedev, and R. Gyllenram, "Towards modelbased closed-loop control of the basic oxygen steelmaking process," in Proc. of the 9th IFAC Symposium on Automation in Mining, Mineral and Metal Processing 1998, Cologne, Germany, 1-3 September 1998, pp. 69-74, 1998.

[5] W. Birk, I. Arvanitidis, P. Jönsson, and A. Medvedev, "Physical modelling and control of dynamic foaming in an LD-converter process," in Proc. of the IEEE Industry Applications Society 35th Annual Meeting, Sheraton Roma Hotel 8-12 October 2000 Rome Italy, 2000.

[6] S. D. Stearns, Digital Signal Analysis. Hayden Book Company, Inc. Rochelle Park, NJ., USA, 2 ed., 1975. Digital signal analysis.

[7] L. Ljung, System Identification Toolbox. The MathWorks Inc., 1995.

[8] S. Skogestad and I. Postlethwaite, Multivariable Feedback Control - Analysis and Design. John Wiley \& Sons, 1996.

[9] A. Johansson, A. Medvedev, and D. Widlund, "Model-based estimation of decarburization rate and carbon content in the basic oxygen steelmaking process," in Proc. of the IEEE Industry Applications Society 35th Annual Meeting, Sheraton Roma Hotel 8-12 October 2000 Rome Italy, 2000. 